\title{
Ruralizando a Dewey: El amigo Americano, la colonización interna y la Escuela de la acción en el México posrevolucionario (1921-1940)
}

\author{
Ruralizing Dewey: The American friend, \\ internal colonization, and the Action School in \\ post-revolutionary Mexico (1921-1940)
}

\section{Ruraliser Dewey : L'ami américain, la colonisation interne, et l'école en action au Mexique post-révolutionnaire (1921-1940)}

\author{
Rosa Bruno-Jofré y Carlos Martínez Valle \\ Queen's University
}

\begin{abstract}
RESUMEN
El artículo examina cómo y en qué medida las ideas de John Dewey fueron adoptadas y adaptadas por la elite educativa y política de México posrevolucionario y sus consecuencias. Provee el contexto político, socio-económico y cultural de la recepción así como las vías de entrada de las ideas de Dewey a México, incluyendo las relaciones entre la Secretaría de Educación Pública, la Universidad Nacional de México y Columbia University (en particular Teachers College), y las misiones protestantes. Discute los problemas e ideas que caracterizaron los debates y mediaron la adopción y adaptación de ideas pedagógicas internacionales y presta particular atención a la construcción de la Mexicanidad (nation building) a través de la incorporación o de la integración (dependiendo de la corriente imperante) de la población indígena y rural en un contradictorio y ecléctico proyecto modernizador. La introducción de las ideas de Dewey se discuten en el contexto de las tendencias educativas y políticas dentro de la Secretaría. No se deja de lado el impacto negativo que tuvieron algunas ideas progresivas en la población rural e indígena. En la última parte, el artículo se centra en la obra de Rafael Ramírez que fue un educador progresivo, un personaje central en la Secretaría donde sirvió por largo tiempo a pesar de los cambio de líderes. Dedicó su vida a la educación rural y su misión civilizadora y dejó un legado escrito que incluye su lectura de Dewey.
\end{abstract}


Descriptores: Dewey; México posrevolucionario; Mexicanidad; Moisés Sáenz; Rafael Ramírez; educación de la población rural e indígena en México; misión civilizadora de la educación.

\section{ABSTRACT}

The article examines how and to what extent the ideas of John Dewey were adopted and adapted by the political and educational elite of post-revolutionary Mexico and the consequences of that adoption. It provides the political, cultural, and socio-economic context of reception as well as the various points of entry of Dewey`s ideas including the relation between the Secretary of Education, the Universidad Nacional de México and Columbia University (in particular Teachers College) and the influence of the Protestant missionaries. The article discusses the issues and ideas that characterized the political and educational debates of the time and mediated the traveling and reception of international pedagogical ideas. It pays particular attention to the building of Mexicanidad (nation building) through the incorporation or the integration (depending on the dominant current thought) of the rural and indigenous population in a contradictory and eclectic modernizing project. It does not neglect the negative consequences of the translation of some progressive ideas. The introduction of Dewey`s ideas are discussed within the context of the various political and educational tendencies inside the Secretary. The last part of the paper is devoted to Rafael Ramírez, a progressive educator, a central protagonist, who served in the Secretary for a long time in spite of political changes and who devoted his life to rural education and its civilizing mission. He wrote about education and we can trace his reading of Dewey.

Key words: Dewey; Post-revolutionary Mexico; Mexicanidad; Moisés Sáenz; Rafael Ramírez; education of the rural and indigenous population in Mexico; education civilizing mission.

\section{RÉSUMÉ}

Cet article examine comment et à quel point les idées de John Dewey furent adoptées et adaptées par l'élite politique et éducative du Mexique post-révolutionnaire et les conséquences de cette adoption. Il fournit le contexte de réception politique, culturel et socio-économique ainsi que les divers points d'entrée des idées de Dewey, y inclus la relation entre le Secrétaire de l'Éducation, la Universidad Nacional de Mexico et l'Université Columbia (en particulier le Collège de l'Éducation), et l'influence des missionnaires protestants. L'article discute des questions et des idées qui caractérisaient les débats politiques et éducatifs du temps et qui véhiculaient les idées pédagogiques internationales. Il porte une attention particulière à la construction de la Mexicanadad (nation mexicaine) par l'incorporation ou l'intégration (dépendant de la pensée courante dominante) de la population rurale et indigène dans un projet de modernisation contradictoire et éclectique. L'auteure ne néglige pas les conséquences négatives de la translation de certaines idées progressistes. L’introduction des idées de Dewey est discutée dans le contexte des diverses tendances éducatives et politiques au sein du Secrétariat. La dernière partie du papier est consacrée à Rafael Ramirez, un éducateur progressiste et protagoniste central, qui servit longtemps au Secrétariat en dépit des changements politiques et qui a consacré sa vie à l'éducation rurale et à sa mission civilisatrice. Ramirez a écrit au sujet de l'éducation et nous pouvons retracer sa lecture de Dewey dans ses écrits.

Mots clés : Dewey, le Mexique post-révolutionnaire, la Mexicanidad, Moisés Saenz, Rafael Ramirez, l'éducation de la population rurale et indigène au Mexique, la mission civilisatrice de l'éducation.

\section{Introducción}

E N el ARTículo Mexico's Educational Renaissance, publicado en The New Republic el 22 de septiembre de 1926, ${ }^{1}$ John Dewey afirmó que la educación rural posrevolucionaria de México, a la que consideraba "uno de los experimentos sociales más importantes que habían tenido lugar en el mundo," ${ }^{2}$ le había devuelto la fe en la 
capacidad de la escuela como instrumento de transformación social y, en definitiva, en sus ideas educativas. El reconocimiento de Dewey de la labor de los educadores mexicanos fue correspondido desde el sur del Río Grande y los homenajes y las declaraciones de deuda con el pensador americano fueron numerosos en el período posrevolucionario. La red de reconocimientos y, por tanto, de mutua legitimación, llegó incluso a la cita recíproca. Así, Dewey citó a Moisés Sáenz diciendo: en ningún lugar he visto un mejor ejemplo de escuela socializada que algunas de las escuelas rurales de México. ${ }^{3}$ Sáenz había sido estudiante en Columbia (1921-1922) y maestro en la Lincoln School de New York. Cuando escribió el artículo Sáenz era Subsecretario de Educación (1925-1931). ${ }^{4}$ Esta unidad del discurso que pareciera implicar la compenetración entre el modelo educativo internacional y su ejecución local, se subraya en otro momento del texto:

He tenido por largo tiempo como idea favorita que los países "atrasados" tienen una mejor oportunidad educacionalmente; que cuando empiezan el camino de la escuela, tienen menos dificultades creadas por la tradición y el institucionalismo que los países donde las escuelas son sostenidas por costumbres que se han endurecido a través de los años. Pero tengo que confesar que nunca he encontrado más evidencia en apoyo de esta creencia que en los países nuevos, educacionalmente nuevos, pueden empezar frescos, con las teorías y prácticas más progresistas de los países educacionalmente más avanzados. ${ }^{5}$

A pesar de que las propuestas de Dewey buscan desarrollar una relación creativa entre la escuela y la sociedad, la cita de Dewey parece olvidar que la interrelación entre educación, política y sociedad transforma el sentido de sus propuestas educativas en contextos distintos a aquellos para los que fueron concebidas. La cita expone la idea de que los países "atrasados," que no cuentan con sistemas y tradiciones educativas establecidas que ofrezcan resistencia al cambio, tienen mayores posibilidades de crear un sistema educativo progresivo. El ahistoricismo de Dewey es consistente con su tendencia a subestimar las condiciones estructurales de la sociedad y las fuerzas políticas, sociales y culturales. Pero, en una escuela tan socializada como la que pretendían crear él y sus "seguidores" mexicanos, la compleja interacción de las ideas educativas con los valores y las fuerzas sociales habría de llevar a la transformación de cualquier proyecto en el momento de su ejecución.

La presencia de Dewey en el discurso pedagógico y los planes educativos del México de los años 20 y 30 parece incontestable, pero es interesante definir esa presencia. Este artículo examina, pues, cómo y en qué medida las ideas de Dewey fueron adoptadas y adaptadas por la élite intelectual y educativa mexicana posrevolucionaria y cómo dieron lugar a efectos perversos al ser aplicadas a realidades distintas a aquélla para las que fueron concebidas. Tras esbozar el contexto político, socioeconómico y cultural de la recepción, pasaremos a estudiar las vías de entrada de las ideas de Dewey a México, incluyendo las relaciones entre la Secretaría (ministerio) de Educación Pública (en adelante, SEP), la Universidad Nacional de México y Columbia University, especialmente Teachers College. Discutiremos algunos de 
los problemas e ideas que determinaron los debates educativos filtrando la adopción y adaptación de las ideas pedagógicas internacionales. En particular, fijaremos la atención en uno de estos problemas centrales, el de la nation building a través de la incorporación/integración de la población indígena y rural en un contradictorio y ecléctico proyecto modernizador. $\mathrm{Al}$ analizar los patrones del discurso educativo y de la recepción de las ideas de Dewey y de otras teorías educativas contemporáneas, haremos referencia a distintas tendencias educativas representadas en la SEP. Empero, centraremos nuestro estudio en la obra de Rafael Ramírez, un alto funcionario de la SEP que, a pesar de los continuos cambios de personal en los años estudiados, permaneció en la Secretaría durante la mayor parte del periodo, convirtiéndose en un personaje central en la institución. Ramírez, un educador progresivo según la definición del momento, dedicó su vida a la educación rural como misión civilizadora para desarrollar la civilización rural.

\section{Contexto}

Las dificultades en crear de México una entidad moral y política son enormes y, a menudo, parecieran insuperables (...) El hecho que los Indios Mexicanos tengan una vida sedentaria de agricultores, una cultura mucho más alta y ofrezcan más resistencia que nuestro propios Indios, sólo aumenta la dificultad de la situación.

Si le agregamos a esto el hecho de que los Indios no son homogéneos entre ellos, están divididos en alrededor de treinta tribus diferentes, son intensamente centrados en sí mismos, celosos de su autonomía, y valoran el aislamiento, que es acentuado por las condiciones geográficas, empezamos a tener una idea del problema que el gobierno pos-revolucionario enfrenta. Y lo hace de una manera tan sistemática como la manera de evitarlos que tenían los regímenes anteriores. ${ }^{6}$

Tras la revolución, México era un mosaico de territorios pobremente conectados en el que las diferencias geográficas y económicas subrayaban su diversidad étnica, política y económica. Así, por ejemplo, se hablaban cientos de dialectos de 72 lenguas pertenecientes a diecinueve familias lingüísticas diferentes. ${ }^{7}$ Apenas siete décadas antes de la revolución México había perdido la mitad de su territorio en la guerra con los USA. Pero además, la Doctrina Monroe o las intervenciones americanas durante la revolución hacían plausible aventurar otras pérdidas territoriales o la degradación del país a mero satélite estadounidense. ${ }^{8}$

La misma revolución era fiel reflejo de esa fragmentación, pues las diferentes guerras civiles que los historiadores de los regímenes posrevolucionarios fundieron en una unidad mítica estaban animadas por muy diferentes problemas, movimientos sociopolíticos y fines. El mínimo común denominador de los movimientos revolucionarios era su reacción a las tensiones políticas, económicas y sociales de la dictadura del General Porfirio Díaz (1876-1911). Ésta se basaba en una economía dependiente 
de exportación de materias primas e importación de manufacturas que requería grandes haciendas, consolidadas a costa de la pequeña propiedad agraria y terrenos comunales y baldíos. Para sostener un desarrollo económico de tan altos costes sociales el régimen se soportaba en redes caciquiles y la alianza con los sectores exportadoresimportadores en el marco formal de una democracia censitaria. Los diferentes movimientos revolucionarios (Maderismo, Villismo Magonismo y Zapatismo) reclamaron tierra o democracia o ambas. Pero, a falta de sólidas definiciones ideológicas y dada su heterogeneidad, estos movimientos no fueron capaces de formular un programa consistente y menos de implantarlo de manera coordinada. En un mundo en el que los carismas, afinidades y redes personales eran tan o más importantes que las ideologías, los diferentes grupos se combatieron entre ellos. ${ }^{9}$

Frente a las más radicales reivindicaciones de democracia o distribución/devolución de tierra, la facción ganadora defendía posiciones moderadas poniendo los acentos en reformas culturales y políticas que soportaban el sistema legitimado en la Constitución de Querétaro (1917). La victoria del constitucionalismo permitió el establecimiento de diferentes gobiernos con un fuerte componente militar. Entre 1920-1934, una serie de generales del estado de Sonora, cuya ideología estaba marcada por un nacionalismo republicano y jacobino en línea con la tradición decimonónica del republicanismo radical más que con las demandas de democracia y reforma agraria radical, conformaron los gobiernos. Esto cambió con la llegada a la presidencia del General Cárdenas (1934-1940) quien, al menos en sus primeros años de gobierno, siguió una política más radical de nacionalismo económico y distribución agraria.

La devastación de la guerra, los conflictos internos que continuaron durante todo el período, la amenaza de los USA y las demandas revolucionarias presionaron a los nuevos gobiernos a consolidar su poder y construir el nuevo estado a través de un proyecto de "reconstrucción" y "modernización" económica y moral e integración socio-política a través de la movilización popular con tonos populistas y nacionalistas. ${ }^{10}$ Este proyecto incluía la desmovilización de los grupos armados, la reforma institucional del estado, una moderada reforma laboral y agrícola y la creación de una plataforma política estable que dirimiese los conflictos entre distintas facciones políticas. El programa incluía la incorporación o integración de la población rural e indígena, no sólo para mejorar sus condiciones de vida, sino también para aculturarlos, en particular a través de la extensión del castellano o "idioma nacional." La Iglesia católica, a la que se responsabilizaba del atraso del país y en particular de la población rural e indígena, fue considerada un poder alternativo al que contener o erradicar. El estado posrevolucionario fomentó no sólo el asociacionismo y cooperativismo, sino también el corporativismo. Éste, fundido con estructuras personales e informales de poder, el cacicazgo, devino clave en la vertebración y articulación del país y en la estabilidad de los gobiernos revolucionarios. Un instrumento central en esta estructura fue la creación de partidos políticos, sindicatos y organizaciones agrarias oficiales u oficiosos que encarnaban una ideología hegemónica. En 1929 se creó el Partido Nacional Revolucionario (NR), predecesor del Partido Revolucionario Institucional (PRI). La educación, en particular la rural, jugó un papel central en el desarrollo y difusión de este proyecto e ideología hegemónica. 
La concepción de la educación pública como agente transformador y adoctrinador llevó a la creación de la Secretaría de Educación Pública (SEP) en 1921, una idea impulsada por José Vasconcelos, quien devino su primer Secretario. La Secretaría reclamó rápidamente una posición central en la escena educativa, aunque la Constitución de 1917 consagraba el "municipio libre" como la unidad organizativa del estado y, por tanto, de su sistema educativo. La federalización de la enseñanza pública pretendía atajar la crisis educativa generada por el cierre de numerosas escuelas que no podían ser financiadas por los magros presupuestos municipales. Pero, como lo han demostrado los trabajos de Rockwell asi como los de Acevedo ${ }^{11}$ para regiones de Tlaxcala y Puebla, muchas comunidades habían mantenido sus centenarias escuelas incluso durante la revolución. En consecuencia, la federalización de la educación en esas regiones supuso la expropiación de las escuelas municipales. Puede argumentarse que la federalización de las escuelas comunitarias significó no sólo la creación de nuevas escuelas, pero también una nacionalización extendida a todo el país de las escuelas comunales, haciéndolas de esta manera agentes representativas del estado y sus proyectos. ${ }^{12}$ Sin embargo, todavía en 1932, la mitad del presupuesto de la Secretaría se gastaba en el distrito y territorios federales (en particular la ciudad de México) mientras que las escuelas rurales eran pobremente financiadas.

Cuatro grandes corrientes pedagógicas estaban presentes en la SEP en el periodo analizado: la que podíamos denominar "espiritualista," encarnada en el pensamiento de José Vasconcelos, Secretario entre 1921 y 1924; la populista desarrollista, representada por el arriba mentado Moisés Sáenz; la socialista, representada por Narciso Bassols, Secretario entre 1931 y 1934 y la anarquista-racionalista, cuyos defensores, como José de la Luz Mena, no alcanzaron los más altos puestos de la Secretaría aunque fue muy influyente en la educación socialista. ${ }^{13}$ Ramírez estaba en línea pedagógica y políticamente con el populismo desarrollista, pero trabajó con los secretarios que personificaban las tres primeras corrientes adecuándose a sus ideas.

Algunas de las ideas centrales que informaron la SEP eran el redencionismo, el reconstruccionismo y el regeneracionismo, que impregnaban no sólo las corrientes pedagógicas sino el discurso político de republicanos, populistas, socialistas, comunistas y, en otra categoría, los protestantes. Estas ideas hicieron que buena parte de la intelligentsia mexicana rechazase el Darwinismo social (Mendelianismo y Weismanismo), como había sido expuesto en la dictadura porfirista, para adoptar las ideas de Lamarck de una estrecha relación entre ontogénesis y filogénesis. Así, al contrario del Darwinismo social, que rechazaba la posibilidad de avance de las "razas inferiores," el Lamarckismo mantenía que la mejora de los individuos se trasmitía al conjunto de la raza, asignando un papel central a los factores sociales y al medio ambiente en el desarrollo de los caracteres biológicos y psicológicos de individuos y razas. ${ }^{14} \mathrm{La}$ adopción del lamarckismo era también una respuesta a los diagnósticos de la psicología social de las etiologías de las sociedades latinas (y por extensión de las criollas e indígenas), que atribuían su atraso frente a las anglosajonas a determinados rasgos del carácter de sus miembros recreados por sus culturas: individualismo, indolencia, falta de espíritu emprendedor, etc. ${ }^{15}$ Todas estas ideas llevaron a enfatizar el poder regenerador de la educación, pero por una escuela que había de transformar no 
sólo el individuo, sino todo el medio social abarcando a toda la comunidad. ${ }^{16}$

Uno de los principales objetivos de esta escuela era, como escribió Vasconcelos en 1923, incorporar la población indígena a la civilización, preparándola para el ejercicio en un futuro próximo de sus funciones como hombres y mujeres, mexicanos y ciudadanos. La “incorporación” de la población rural e indígena se entendió como la transformación de la cultura de las comunidades rurales siguiendo los estándares de la minoría blanca. Pero este proyecto, en boga en los primeros años 20, fue paulatinamente sustituido por la "integración” de las masas indígenas y campesinas y de México, ${ }^{17}$ un paradigma que afirmaba que la cultura y carácter indígenas tenían rasgos positivos que debían ser preservados e incluso extendidos al resto de los mexicanos en su evolución hacia la integración del país. El cambio de paradigma respondió al fallo de las políticas incorporacionistas, evidente con la crisis económica de 1927, y al mejor conocimiento de las culturas indígenas por los agentes de la SEP.

La necesidad de transformar el medio social para llevar a la práctica el discurso de una mexicanidad regenerada demandaba una idea muy amplia de educación que incluyese diversos instrumentos de pedagogía informal tales como el arte y rituals y celebraciones públicas para generar la participación de toda la communidad. Algunos de estos instrumentos, en particular en zonas rurales fueron las Casas del pueblo (escuelas con muy amplias atribuciones), las Misiones culturales, las Brigadas médicas, las Bibliotecas ambulantes o los Carros escuela (escuelas en vagones de tren). Sus objetivos eran también omnicomprensivos e incluían la salud, la vida doméstica, la mejora de las técnicas agrarias, la recreación y la vida social o la extensión de la cultura en el medio campesino, todos ellos necesarios para el progreso de la nación. Para sustentar las iniciativas de la SEP e involucrar a la población en la transformación de su medio ambiente, impidiendo el retroceso a etapas menos desarrolladas, la SEP promovió la creación de todo tipo de asociaciones. Las promovidas por una Misión cultural pueden servir de ejemplo: "Comisión para la mejora de las condiciones de vida, una Comisión de caminos, la Sociedad de mujeres y madres, la Cruz Roja local, el Grupo de teatro, el Club deportivo infantil, la Campaña antialcohólica." ${ }^{18}$

\section{Las vías de entrada de las ideas de Dewey y la Nueva Educación}

Siguiendo este objetivo de crear una escuela redencionista-regeneracionista que debía transformar cuerpos y espíritus, cambiar hábitos y creencias y fomentar la cooperación e identificación con las nuevas instituciones nacionales, los líderes educativos mexicanos intentaron desde temprano desarrollar instrumentos pedagógicos propios y seleccionar ideas internacionales que pudieran servir a sus necesidades. La idiosincrática y ecléctica pedagogía que se fue conformando se denominó Escuela de la acción. Así, como muchos países latinoamericanos, el México posrevolucionario estuvo abierto a todas las ideas de la moderna psicología y la renovación pedagógica, de Kropotkin a Decroly, de la pedagogía revolucionaria soviética, ${ }^{19}$ a Ferrière o el mismo Dewey. Aunque, como manifestó Rafael Ramírez, la Escuela Nueva era un movimiento importante, pero "en esencia no trascendental." ${ }^{20}$ Como argumentos políticos, las valoraciones sobre la influencia extranjera en la pedagogía mexicana eran 
muchas veces contradictorias, pero se tendió a ver estas ideas como refuerzos para el desarrollo de una pedagogía idiosincráticamente mexicana que estaba adquiriendo sus rasgos y contenidos a través de la práctica. Así, la importación de ideas fue considerada más como una "feliz inserción" que creaba un mestizaje espiritual que como un simple trasplante. ${ }^{21}$

El pensamiento de Dewey, enraizado en su propio tiempo, poseía algunos elementos que podían atraer a los líderes mexicanos. Ambos compartían el lenguaje, dominante en los meliorismos sociales y social gospelers americanos, del reconstruccionismo y redencionismo que consideraba a la escuela como la principal fuerza de cambio social. La Escuela de la acción que, a pesar de sus eclécticas influencias e idiosincráticas características estaba alineada con algunas ideas de Dewey, fue considerada por muchos como el instrumento más adecuado para alcanzar el ideal de la nueva mexicanidad. Como escribió Francisco Arze Gurza, la “integración” de escuela y vida defendida en la escuela activa no se consideraba diferente de los proyectos de integración cultural y social del mosaico mexicano que eran, para Vasconcelos o Sáenz, la base para la construcción de la nación mexicana. ${ }^{22} \mathrm{El}$ nacionalismo y la integración social eran los principales filtros de la reinterpretación o adaptación de toda idea pedagógica foránea, aunque no los únicos.

A pesar del lugar común, ${ }^{23}$ parece que Dewey sólo tuvo un papel reducido en la primera definición de la Escuela de la acción mexicana como quedó plasmada en las Bases para la organización de la escuela primaria conforme a los principios de la acción, aprobadas por Vasconcelos en 1923 para el Distrito y los Territorios Federales. El grupo de inspectores de zona que conformaban el Consejo Técnico del Departamento Escolar del Distrito Federal, responsable de la redacción de las Bases, estaban más interesados en la obra de Ferrière, Decroly o Kropotkine (sic). Siguiendo los vínculos tradicionales de la intelligentsia mexicana con la cultura francófona, Eulalia Guzmán, ${ }^{24}$ quien escribió La escuela nueva o de la acción, el más claro precedente de las Bases, visitó algunas escuelas mexicanas (Bacum) y de los USA, pero su principal inspiración parece ser, según Larroyo, Decroly y sus centros de interés ${ }^{25}$ y, según Fell, Ferrière, a cuyas clases asistió Guzmán en Suiza. De Ferrière, Guzmán habría tomado la valoración de Dewey como psicólogo educativo que se repetirá en las obras de Rafael Ramírez. ${ }^{26}$

Pero las menciones a Dewey en el discurso pedagógico se incrementan rápidamente. Ya lo había citado el profesor Lisandro Calderón en el Primer Congreso Mexicano del Niño en 1921. ${ }^{27}$ Salvador M. Lima y Marcelino Rentería del grupo de educadores e inspectores de zona que redactaron las Bases y que tenía como órgano oficioso la revista Educación afirman en ésta en 1923 que los principios de la escuela activa, como movimiento global, fueron enunciados por Decroly, Ferrière, Kerschensteiner y Dewey. ${ }^{28}$ Entre los pedagogos de la Escuela nueva mencionados por un funcionario relacionado con el Plan de trabajo de las escuelas rurales federales de 1925 citado por Ramírez están J. F. Eslander; ${ }^{29}$ Faria de Vasconcelos; ${ }^{30}$ Angelo Patri, un graduado de Columbia influido por Dewey ${ }^{31}$ y John Dewey, en particular su School and Society ${ }^{32}$ traducida por Domingo Barnés ligado a la Institución libre de enseñanza. ${ }^{33}$ Moisés Sáenz en la clase magistral dada en la Universidad de Chicago, en 
el verano de 1926, que Dewey cita arriba, dijo que School and Society "es conocida y querida" en México y que las ideas deweyanas se habían puesto en práctica completamente en México. Menciona como conceptos deweyanos que señalan una nueva tendencia en educación: motivación, respeto a la personalidad del niño, autoexpresión, vitalización del trabajo escolar, método de proyectos, learning by doing, democracia y educación. Sáenz llego a afirmar, hiperbólico, que sin querer Dewey había metido a México en una "guerra pedagógica" que enfrentaba a sus seguidores, los liberales, con los reaccionarios. ${ }^{34}$

Pero, a pesar de que tradicionalmente se considera a Sáenz como uno de los conocedores e introductores de Dewey en México, hay diferencias claras entre las propuestas de ambos. Por ejemplo, el proyecto de Carapan (1932) no trasluce un conocimiento y aplicación del método científico en el sentido deweyano del ejercicio de crítica sistemática aplicada a la intelección empírica. Pero, no sólo no hubo un diseño claro en Carapan. Sáenz también abogó por separar la práctica educativa y la investigación rechazando las concepciones deweyanas de la relevancia de la interrelación entre teoría y práctica, idea y acción. Como el principio redencionista implicaba la imposición de los elementos salvíficos, no hubo espacio para que los estudiantes se involucrasen en un proceso de reflexión experimental auto-correctora. El redencionismo le hizo también apoyar el uso de instrumentos educativos memorísticos (catecismo cívico revolucionario) o irracionales emocionales (ritos, celebraciones) para la propaganda de un espíritu nacional que compara a las religiones nacionales del comunismo o los fascismos. El trabajo de Sáenz en Carapan no muestra conocimiento o aceptación de las propuestas deweyanas de la imbricación entre democracia y educación y, a pesar de que rechaza las nuevas estructuras caciquiles surgidas de la revolución, se sirve de ellas en su proyecto civilizador. Sáenz llegó a afirmar que la carretera como medio de comunicación sería, más que la escuela, el principal agente de la integración y modernización de México. ${ }^{35}$

En la evolución de la percepción de la obra de Dewey es interesante un artículo aparecido en un órgano oficial de la SEP, El Maestro Rural en 1933. "Acaso, dice la revista, no haya un nombre que resuene más a los oídos de nuestros maestros que el de Dewey," pues se asocia "en particular, a su sistema de proyectos (sic) en nuestra pedagogía contemporánea." El artículo asocia equivocadamente Dewey a la psicología de Thorndike y de Stanley Hall e incluso dice que Dewey le imprime a estas concepciones un sello nuevo al introducir el concepto de "obra democratizadora o socializadora." Se asevera también que Dewey no creía ni en la metafísica ni en el pensamiento desinteresado y que este pragmatismo estaba en la base de su ciencia pedagógica. Pero el artículo diferencia el pragmatismo de Dewey de un materialismo cruel, pues está balanceado por un idealismo objetivo. ${ }^{36}$ Esta distinction parece ser ajena a Dewey.

Los escritos de Moisés Sáenz o Rafael Ramírez muestran que en México hubo un acercamiento funcional a la educación progresiva y a las teorías de Dewey. Este acercamiento enfatizaba los aspectos pedagógicos de su obra desgajándolos de su filosofía. Esto se debía en parte a que la mayoría de sus introductores eran normalistas que habían ampliado sus estudios de pedagogía, por lo que sólo tenían un conocimiento 
y comprensión limitados de los aspectos filosóficos de su obra. A este entendimiento reductivo contribuyó la popularidad de Edouard Claparède, que medió la intelección de Dewey en Iberoamérica. ${ }^{37}$ En 1926, el año en el que Dewey visitó México, la Editora franco-americana de México publicó como obra independiente el prólogo de L'école et l'enfant de Claparède, titulado "La pedagogía de John Dewey," que ya había sido traducido al español en 1922 en el Boletín de la Institución Libre de Enseñanza. ${ }^{38}$ En él, Claparède separa la filosofía de Dewey de su teoría educativa, de forma que mantiene que su psicopedagogía podía ser utilizada sin atender al pragmatismo. No se puede dejar de lado tampoco el hecho de que el grueso de las obras filosóficas de Dewey no fue traducido y publicado en México hasta fines de los años cuarenta, principios de los cincuenta, cuando el Fondo de Cultura Económica publicó las traducciones de Gaos, Imaz o Ramos. ${ }^{39}$

En la interpretación de Dewey también mediaron Botroux y Bergson. Así, Vasconcelos hizo suya la crítica a la ciencia de los franceses y la aplicó en particular al Darwinismo, al que consideraba una ideología colonial. ${ }^{40}$ Dentro de esta línea de pensamiento, Vasconcelos que, aunque renuente, apoyó la socializadora Escuela de la acción como alternativa a la escuela tradicional y la individualista liberal, desarrolló una gran aprensión a las ideas de Dewey y al pragmatismo. Vasconcelos consideró especialmente peligroso el énfasis en aprender haciendo (learning by doing) y la noción mal entendida de la escuela como instrumento para la adaptación al medio ambiente que, desde su punto de vista, crearía una escuela adaptadora en vez de liberadora. Esta interpretación refleja una lectura reductiva de Dewey ya que la pedagogía de Dewey entendía a la educación como un proceso experimental transformador. Esta crítica retrospectiva apareció fundamentalmente en De Robinson a Odiseo publicado en 1935, después del fracaso de su candidatura a la presidencia mexicana, que él atribuyó a la influencia americana y sus aliados protestantes. ${ }^{41}$ La crítica no sólo se basaba en su espiritualismo, la búsqueda de una regeneración por la estética sino también en el cuestionamiento del utilitarismo e imperialismo americano. Dewey se había convertido en un instrumento de legitimación de las ideas de los populistas-desarrollistas (entre ellos Sáenz) frente al espiritualismo vasconceliano.

Especialmente durante los años 20 la "presencia" de Dewey en la escena políticoeducativa mexicana tuvo una dimensión institucional. Hubo una relación sostenida de la SEP y un número de educadores mexicanos con la Universidad de Columbia y Teachers College. En abril de 1928, Rafael Ramírez, Director de Misiones Culturales, visitó Nueva York comisionado por el Secretario Puig Casauranc para participar en una Conference on Education en Teachers College y reconocer públicamente la contribución de la Universidad de Columbia a la educación mexicana. Ramírez tituló su presentacion What is the American Contribution to the Educational Progress of the World? En ella afirma que México es un país con fuertes tradiciones y es difícil transformar los valores nacionales, pero reconoce la inspiración y el apoyo de los maestros norteamericanos. Así, Ramírez reiteró que un número de educadores mexicanos graduados en Columbia (por ejemplo Manuel Barranco, ${ }^{42}$ Moisés Sáenz, Manuel Gamio $^{43}$ o Daniel Cosío Villegas ${ }^{44}$ ) ocupaban puestos de responsabilidad, inspirando y conformando la educación mexicana. Especial mención le merece el curso sobre 
educación secundaria organizado por el International Institute of Education para maestros mexicanos en el invierno de 1925, que, añade, tuvo una gran relevancia para la reorganización de la educación secundaria mexicana, aunque ésta mantuviera sus especificidades. Ramírez continuó su recuento de las conexiones con Teachers College refiriéndose a los cursos de verano de la Universidad Nacional de México, que, entre otros, había invitado a Dewey a dar dos cursos: "Contemporary Philosophic Thought" y "Advanced Educational Problems" 45 en su sexta convocatoria, del 5 de julio al 21 de agosto de $1926 .{ }^{46}$ Rafael Ramírez también se refería a la firma de convenios de colaboración y a la comunicación con profesores americanos cercanos a Dewey, como el Dr. Kandel del International Institute del Teachers College y Frank Tannenbaum que estudiaron el sistema educativo mexicano. ${ }^{47}$ La visita de Dewey estuvo precedida por discursos pronunciados por Puig Casauranc, Secretario de la SEP entre 1924 y 1928 y entre 1930 y 1932, en Columbia el 23 y 24 de marzo de 1926.

En 1934 la SEP distribuía a precio módico los siguientes títulos de traducciones de escritos de Dewey incluidas en la colección Obras pedagógicas de consulta: "Ensayos de educación"; "Teoría sobre la educación”(Democracia y Educación); "Cómo pensamos"; "Filosofía de la educación. Los valores educativos"; "Los fines, materias y métodos de la educación"; "El hábito y el impulso en la conducta: la inteligencia y la conducta"; "Reconstrucción de la filosofía"; "Las escuelas de mañana"; "Pedagogía y filosofía"; "El niño y el programa escolar"; "El interés y el esfuerzo en educación." Otra vía de acceso a Dewey fueron las escuelas americanas protestantes. En 1885 había en México 80 escuelas elementales con 3.000 niños, que, siguiendo el crecimiento de los alumnos, se extendieron a numerosas escuelas secundarias. Como escribió Bastian, la escuela era un instrumento fundamental en el trabajo misionero. Los recién convertidos ministros metodistas o presbiterianos devinieron maestros y divulgadores de "valores modernos ligados a la prédica del evangelio," incluyendo los deportes. Estas escuelas bilingües daban a los hijos de los conversos la posibilidad de recibir valores "modernos" embebidos en una nueva pedagogía y fomentaron la movilidad social. ${ }^{48}$ Las escuelas protestantes introdujeron métodos pedagógicos innovadores, enseñaron habilidades industriales y comerciales, fomentaron la resolución de problemas y transmitieron valores culturales de los USA. ${ }^{49}$ Desde fines del siglo XIX y más claramente en el XX, las escuelas protestantes transportaron la influencia del Social Gospel y su reformismo así como una pedagogía basada en la educación progresiva (progressive education).

La influencia de la última y particularmente de John Dewey, era patente a través de la obra de George Coe, educador religioso protestante cuya obra llegó a las escuelas misioneras, y a quien Donald Meyer definió como "el más capaz de los defensores de las ideas progresivas,... un estudioso de John Dewey." 50

La presencia protestante en el México (post)revolucionario es significativa. Los protestantes, que consideraban a la Iglesia católica como un enemigo y un impedimento para el progreso, apoyaron la revolución y los gobiernos posrevolucionarios, a pesar de que también tuvieron que sujetarse a las leyes que restringían la educación religiosa en sus propias escuelas. Hubo protestantes en puestos prominentes de las administraciones posrevolucionarias, como el presbiteriano Sáenz. ${ }^{51}$ Bajo su dirección, 
la SEP continúo desarrollando la educación rural siguiendo el modelo de la Escuela de la acción. De acuerdo a Miller, durante su mandato las escuelas rurales pasaron de 300 a 6800 y el número de sus estudiantes de 18,0000 a 600,000. Los católicos se quejaron de que muchos de los maestros reclutados para las escuelas rurales provenían de escuelas protestantes. ${ }^{52}$ Hubo, también, contactos entre miembros de la SEP y protestantes reformistas. Así, incluso José Vasconcelos fue miembro del consejo editorial de La Nueva Democracia (1920-1964), una revista de opinión con una perspectiva evangélica, publicada por el Inter-Denominational Committee on Cooperation in Latin America (1913), que a su vez tenía una estrecha relación con Teachers College. En el congreso que organizó en Panamá, "Christian Work in Latin America," participaron tres profesores del Teachers College: Paul Monroe y James E. Russell, decano en funciones y su sucesor y T.H. P. Sailer. Los tres eran claramente reconstruccionistas y conocedores de la educación progresiva. ${ }^{53}$ Curiosamente, el Secretario del Committee, Samuel Guy Imman era un defensor del Social Gospel y del Panamericanismo, una doctrina que tanto Vasconcelos con su enfoque espiritualista y los Arielistas, asì como el izquierdismo rechazaban como imperialista. ${ }^{54}$

Pero el papel de Dewey como icono de la modernidad educativa y legitimador externo de políticas internas, que alcanzó su máximo con la preponderancia de la corriente populista desarrollista, fue declinando con la introducción de la educación socialista. ${ }^{55}$ Ésta fue establecida oficialmente con la reforma del artículo 3 de la Constitución en 1934, bajo los auspicios de Narciso Bassols, Secretario de Educación entre 1931y 1934. Éste subrayó las funciones sociales de la escuela, el desarrollo de la conciencia de clase, el materialismo, racionalismo y su misión desfanaticizadora. Así, las referencias a Dewey son menos frecuentes en este período mientras que aumentan las de los pedagogos soviéticos. Pero dada la falta de los necesarios desarrollos pedagógicos y didácticos para conducir la práctica educativa y a la vista del percibido fallo del método de complejos soviético, ${ }^{56}$ se continuaron usando los métodos e instrumentos de la Escuela de la acción.

\section{Rafael Ramírez y sus lecturas de Dewey}

Rafael Ramírez fue una figura central en la SEP. Tras graduarse en la Escuela Normal de Jalapa, que había dirigido E. Rebsamen y era una de las más prestigiosas del país, trabajó como maestro rural y se trasladó al Distrito Federal a enseñar Metodología en la Normal Nocturna. En 1921 inició su carrera como inspector en la SEP bajo Vasconcelos, en 1923 fue nombrado Director de la primera Misión cultural en Zacualtipan y, en 1927, Director del Departamento de Escuelas Rurales, Primarias Foráneas e Incorporación Indígena. Cárdenas le nombró en 1934 Consejero del Departamento de Escuelas Normales Rurales y Agrícolas, una posición que combinó con labores de dirección y profesorado en escuelas normales. ${ }^{57}$

Junto con el Secretario Puig Casauranc, Ramírez defendió firmemente la federalización de la enseñanza en el Primer Congreso del Niño mantenido en México D.F. en enero de $1921^{58}$ (Meneses 389). También fue paladín de la incorporación de los aborígenes, en particular a través de la adopción del castellano, el "lenguaje nacional," como 
medio para construir el nuevo México. Empero, a inicios de los 30, Ramírez abrazó la integración y, paralelamente, trabajó en la implantación, definición y divulgación de la escuela socialista siguiendo la política de la SEP del momento, lo que muestra el eclecticismo que caracterizaba la SEP y el conjunto de la escena educativa.

En el invierno de 1925, Ramírez participó en el curso sobre educación secundaria organizado por Teachers College de Columbia para maestros mexicanos citado arriba. Plausiblemente asistió a los cursos de Dewey en México en 1926 y en la primavera de 1928, Puig y Sáenz le pensionaron para que estudiase la educación rural en los USA. Para la planificación del viaje y las cartas de recomendación contó con la ayuda del Teachers College y en particular de Mabel Carney del Departamento de Educación Rural. Empero, a pesar de su exposición a la educación americana y a las ideas internacionales y de su discurso de 1925 en Columbia, Ramírez afirmó que: "uno se da cuenta de que la doctrina mexicana de la educación rural es más certera y por lo tanto más eficaz, y de que tal doctrina merece cumplidamente el título de educación rural mexicana, porque todos sus rasgos característicos son propios y no ajenos." 59

La primera mención a Dewey en la obra de Ramírez aparece en 1915 en relación a la educación industrial, un instrumento necesario "para regenerar a nuestro pueblo enfermo de indolencia." ${ }^{60}$ La educación industrial fue su foco de atención hasta que en la misión cultural de Zacualtipan desarrolló su interés por la educación rural que devino el centro de su carrera. El texto en el que Ramírez menciona a Dewey es una cita parafraseada de una obra de Omer Buyse sobre las escuelas industriales de los USA. Buyse menciona las escuelas experimentales elementales afiliadas la Universidad de Chicago o el Teachers College de la Universidad de Columbia "donde los actos de los niños, orientados por una idea muy atrevida, llegan a ser la base de toda la enseñanza, pasando la acción del maestro al segundo plan (sic)." Un comentario este que parece implicar un entendimiento deficiente y simplificado del papel del maestro como guía del proceso de investigación que caracterizaba la escuela progresiva en particular, la influenciada por Dewey.

La referencia a Dewey como profesor de Pedagogía y no de Filosofía, puede ser leída como un primer filtro y adaptación a las tradicionales divisiones disciplinarias mexicanas (y de la francofonía). Pero más importante es la errónea atribución a Dewey que realiza Ramírez, usando a Buyse como fuente, ${ }^{61}$ de la teoría de la recapitulación y, específicamente, de la teoría de las épocas culturales en el sentido de que la evolución del niño reproduce la de la humanidad. El texto continúa afirmando que los instintos del hombre primitivo se encuentran en el niño, lo que hace que este se interese en ver y manipular las cosas con las que vivieron sus ancestros. La misión de la escuela es, entonces, replicar la evolución humana haciendo que el estudiante pase por las fases de conciencia que las razas superiores han recorrido hasta alcanzar su estado civilizatorio superior. ${ }^{62}$ La cita de Buyse es problemática pues la aplicación a la pedagogía y el currículo de la teoría de las épocas culturales fue promovida por discípulos alemanes y americanos de Herbart y tiene un defensor fundamental en Stanley Hall. ${ }^{63}$ Dewey criticó esta teoría en Democracy and Education, pues para él es el presente el que genera los problemas que pueden guiarnos al pasado, por lo que la cita no hace justicia a sus concepciones. ${ }^{64}$ 
Ramírez demuestra en sus obras de los 20 y los primeros 30 una cierta familiaridad con las ideas pedagógicas deweyanas, pero esta no parece estar enraizada en el conocimiento del pragmatismo y presenta rasgos eclécticos. Ramírez concibe una escuela rural moderna informada por un espíritu progresivo que incluye a niños y adultos, la mejora de la comunidad y tiene como fin la promoción del progreso de la civilización rural. Ramírez coloca a la comunidad en el centro de su pedagogía, pero es difícil afirmar que haya tomado ese concepto de Dewey, aunque School and Society circulaba por México. La noción deweyana de la comunidad como organismo constituido por experiencias, sentidos, decisiones y valores compartidos que conforman significados a través de la comunicación simbólica se oponía al individualismo liberal y tenía connotaciones comunitaristas. La lengua y la cultura son centrales en el entendimiento de una comunidad orgánica.

Ramírez acepta una concepción orgánica de la sociedad pero la cultura y la lengua indígena son vistas como disfuncionales en el proyecto de comunidad que propicia. En 1933, Ramírez escribió un artículo en "El Maestro Rural” sobre la incorporación de los aborígenes en la nueva mexicanidad a través de la enseñanza del castellano. Consideraba que el castellano era instrumental para adquirir otros conocimientos, de modo que los niños indios debían ser enseñados en castellano para que se comunicasen con "nosotros." La noción de otredad permea el acercamiento pues estaba en el centro del proyecto de la incorporación de la "raza indígena" a la "nuestra." Para Ramírez no existían motivos prácticos para aprender los lenguajes de los indígenas, por lo que ellos habrían de aprender castellano. Pero además, siguiendo las concepciones lamarckianas del ambiente como formador de la personalidad, advertía al maestro que no usase en la comunicación con los discentes los idiomas indígenas pues esto lo llevaría a aprender la lengua indígena y correría el riesgo de ser asimilado a las costumbres indígenas y sus formas inferiores de vida. El maestro se convertiría él mismo en un "indio," objeto de reincorporación o reeducación. La enseñanza del castellano habría de transformar a los indios en "gente de razón," la denominación tradicional para hispanos y criollos. Esta concepción del lenguaje como el medio de introducir en las comunidades indígenas "nuestras costumbres y formas de vida, que indudablemente son superiores a las suyas," esto es, como base de la personalidad y fundación de la cultura y el proyecto nacionales, es más propia de los nacionalismos románticos del XIX que del estado democrático participativo del XX. ${ }^{65}$ La incorporación y el redencionismo en el que se justificaba, llevaban implícitos la negación de la cultura del educando, pues para justificarse, tenían, como precondición, que negar discursivamente las cualidades de la cultura del redimido. ${ }^{66}$ La continua referencia a los misioneros del S. XVI en el discurso educativo mexicano del periodo subraya los paralelismos de las misiones y colonizaciones de la edad moderna y contemporánea. ${ }^{67}$

Ramírez acentúa la importancia de enseñar castellano a los niños indígenas "como Dios manda," impidiendo la traducción entre la lengua materna y la enseñada. Para ello se ha de colocar a los niños en situaciones vitales que les conduzcan a aprender el nuevo lenguaje de forma natural, por la experiencia, de igual manera a como aprendieron el materno. Para ello el maestro habría de cuidar los impulsos sociales del niño que le movían a expresarse, facilitando la sociabilidad entre los alumnos y 
subordinando otros aspectos escolares, como el mantenimiento de los horarios, a este fin. Además, para impedir la involución recordaba al maestro que no sólo lo era de los niños, sino de toda la comunidad. La Escuela de la acción devino una tecnología para la aculturación y fue vista como un instrumento para forzar la "modernización" a través de la inmersión en experiencias conducentes a "estadios de cultura superiores." ${ }^{8}$ Este tipo de educación ofrecía la justificación para la expansión del control educativo a todo el medio ambiente y la penetración en ámbitos vitales previamente reservados a la familia o el individuo, como la higiene y la sexualidad, para modelar al nuevo mexicano. No es extraño pues que, por ejemplo, en Carapan los indígenas increparan a Moisés Sáenz que no era su padre. ${ }^{69}$

El pensamiento de Ramírez cambió a la par que los discursos educativos mexicanos. En las conferencias sobre la escuela socialista de 1935 impartidas a inspectores de la SEP ya mantenía, pero sin hacer referencia a México o su propia obra, que las escuelas eran instrumentos del colonialismo europeo y USA para destruir las culturas indígenas y crear otra nueva, "que se podía vivir como otra cadena" de las muchas impuestas por estos sistemas. ${ }^{70} \mathrm{Al}$ contrario de Dewey quien se apropió del Darwinismo como un instrumento filosófico que le sirvió para desafiar distinciones artificiales entre idealismo y empirismo y, entre otras cosas, para desarrollar su teoría de la naturaleza humana, Ramírez, tanto como Vasconcelos, vió en el Darwinismo una ideología colonial de dominio impuesta en México por el Porfiriato y su aliado el capital internacional.

Ramírez definió la escuela socialista como nacionalista, igualitaria, antifanática y científico-materialista. Según Ramírez, como escuela socializada no se diferencia de su ambiente, pues es un instrumento para la mejora social, para generar la conciencia colectiva en los estudiantes y enseñarles a organizarse de forma colectiva para alcanzar sus objetivos. Sin embargo, aclara que este acercamiento no significa que la escuela tuviera que estar organizada como una democracia ni ser un laboratorio de la huelga. ${ }^{71}$ Pero Ramírez no consideraba que hubiese grandes diferencias con los programas anteriores pues la escuela rural mexicana "está empeñando a los niños en formas de trabajo socialmente útiles casi con exclusión de cualquier otra actividad.”72

Esta afirmación contrasta con su distanciamiento de la Escuela de la acción en la que la socialización era un componente fundamental. En esta época, Ramírez asimilaba la Escuela de la acción a los centros de atención o de acción (sic) que atribuía a Dewey, que, más que un proyecto educativo global, constituían, a su parecer, sólo una nueva forma de ordenar el programa escolar. ${ }^{73} \mathrm{El}$ acento en los aspectos no relacionados con el currículo tradicional, había llevado, a su parecer a una serie de disfunciones como la falta de horarios, la vocacionalización y rudimentarización de la enseñanza y el descuido de la lectura, escritura y matemática básica. ${ }^{74} \mathrm{~A}$ pesar de su aprobación de la escuela socializada, Ramírez también vio esos peligros y afirmó que el niño del medio rural tiene el mismo derecho a una educación no meramente vocacional, equilibrada y alfabetizada, así como el acceso a la secundaria y la universidad que garantizaban el ascenso en la escala educativa. ${ }^{75}$

Pero estos no fueron los únicos efectos perversos de la introducción de la Escuela de la acción. A pesar de la federalización, la SEP corría muchas veces sólo con los 
gastos corrientes de material educativo básico y el maestro. Así, dado que las exigencias de la Escuela de la acción en terrenos, edificios (jardín, huerto, teatro, campos de deportes, teatros, cuadras), aperos, plantas y animales, eran muy superiores a las de la escuela tradicional, aquella se convirtió en una carga onerosa para las comunidades. Las demandas de la escuela federalizada pudieron ser concebidas, entonces, como la continuación de sevicias tradicionales que se habían de prestar en monetario o especie para trabajos comunales o del cacique. ${ }^{76}$ Pero era el trabajo infantil en la Escuela de la acción el que concitaba un rechazo especial en las comunidades, pues estas pensaban que la escuela o el maestro arrebatan a la familia esta parte importante de la economía doméstica. ${ }^{77}$ Así, si bien la Escuela de la acción podía ser un instrumento válido para el proletariado rural que también había perdido sus instrumentos de producción y socialización, no lo era tanto para las zonas de pequeña propiedad. Desde la óptica de las comunidades, la asistencia a la escuela se justificaba porque transmitía un conocimiento, la alfabetización, que las familias no podían ofrecer a sus retoños, mientras que la Escuela de la acción pretendía enseñarles justamente aquellos conocimientos que constituían el centro de la cultura y vida familiar, lo que aumentaba la sensación de enajenación del niño. La Escuela de la acción iba a contracorriente de las concepciones tradicionales que la consideraban el medio de la movilidad social que permitía liberarse del trabajo manual, no el instrumento para fijar los individuos a una tierra que apenas podía alimentarlos. Además, en su afán por modelar el medio ambiente, la Escuela de la acción no sólo invadió espacios como la religión, la moral o la higiene que, según las percepciones de la población rural no le correspondían, sino que polarizaba e incrementaba el faccionalismo al interior de las comunidades.

Rafael Ramírez muestra una gran familiaridad con las ideas pedagógicas de Dewey en el opúsculo titulado "Los nuevos rumbos de la didáctica." Pero significativamente la primera edición de la que tenemos constancia es de 1950 y apareció en el n. 14 (mayo) de Revindicación, la revista del Sindicato Nacional de Trabajadores de la Educación, cuando Ramírez estaba apartado de la política y la administración educativa (y por lo tanto tuvo tiempo para leer) y cuando Dewey no podía ya ser considerado como un nuevo rumbo en la didáctica. ${ }^{78} \mathrm{El}$ texto perfila las nociones del aprendizaje cooperativo a través de la experiencia, el desarrollo de hábitos y disposiciones, la reconstrucción y expansión de las experiencias (building on experience). Incluso delinea las fases de la teoría de la investigación (inquiry) que aplicaba a la resolución de problemas y que describe como una forma de acción del pensamiento. ${ }^{79}$ Empero, no se refiere a la noción de investigación sistemática en relación con la construcción de una sociedad democrática auto-correctora, que es central a las teorías deweyanas de la educación y de la democracia. De hecho, la systematic inquiry habría puesto límites al adoctrinamiento y modernización que pretendía la educación posrevolucionaria. En la práctica, la escuela rural, tan cara a Ramírez encarnaba contradicciones, enraizadas en conceptos redentoristas de educación, que podrían ser consideradas como un intento de colonización interna. 


\section{Conclusión}

Líderes y educadores mexicanos del periodo postrevolucionario crearon una noción ecléctica de nueva educación que se decantó en la práctica en la Escuela de la acción. Esta devino una tecnología o instrumento para redimir e incorporar/integrar a la población indígena y rural al nuevo concepto de nacionalidad a través de la formación de los niños y la reconstrucción o regeneración del medio social para hacerle educativo permeándole de los alternativos valores posrevolucionarios. La Escuela de la acción ofrecía los medios y la justificación teórica para penetrar en todos los aspectos de la vida de los individuos y las comunidades, especialmente aquellos más difíciles de alcanzar para la escuela tradicional, desde la producción agraria, a la higiene, la vida domestica o la religión. La Escuela de la acción fue un instrumento para avanzar en, según palabras de Calles, la revolución de las conciencias.

Los presupuestos y objetivos específicamente mexicanos del proyecto educativo posrevolucionario hicieron que sus diseñadores fueran selectivos en la importación de ideas educativas foráneas. Así, y a pesar de la propia presencia de Dewey en México y las estrechas relaciones con Columbia University, sus ideas no aparecen netamente separadas de otros pedagogos de la nueva educación. Dewey aparece mencionado y entendido, muchas veces errónea o reductivamente, en relación a ideas de Kilpatrick, Decroly o Ferrière, Haller, Kropotkin, Lunacharski o el método de complejos.

En este proceso de adopción selectiva, los educadores mexicanos separaron, siguiendo a Claparède (véase en este volumen Bruno-Jofre y Jover), la pedagogía de Dewey de su filosofía y, con ella, de las reflexiones sobre la democracia. Se interesaron por aquellas ideas de Dewey que podían instrumentalizar en su proyecto de regeneración del país y redención de la población indígena y rural. Esto es, entendieron y transformaron a Dewey como un educador para la construcción de la Mexicanidad y la incorporación/integración social. El aspecto en el que más claramente se muestra la existencia de este filtro que acomodó la pedagogía deweyana a las necesidades mexicanas es en la ruralización de un modelo educativo que había sido diseñado para unas condiciones sociales, como las de los USA., en las que la industrialización había arrebatado a las familias los medios de producción en los que anteriormente se educaban y socializaban los niños, por lo que esta tarea había de ser cubierta por la escuela. ${ }^{80}$ Así, a pesar de que la Escuela de la acción fue propuesta inicialmente como un instrumento regenerador del urbano Distrito Federal, se aplicó a la educación rural sin evaluar el impacto y los efectos perversos que su aplicación podía generar, incluyendo entre otros la resistencia de la población rural.

\section{Notas}

1. Este es uno de los tres ensayos de Dewey sobre México publicados por New Republic. Los otros dos ensayos son "Church and State in Mexico" y "From a Mexican Notebook." Fueron reeditados en "Impressions of Soviet Russia and the Revolutionary World: MexicoChina-Turkey." Este artículo fue enviado por el tesorero de la revista al presidente Calvin Coolidge para pedir comprensión y simpatía hacia México. 
2. John Dewey, "Mexico's Educational Renaissance" en: John Dewey, The Later Works, 1925-1953, Vol. 2: 1925-1927 (Carbondale: Southern Illinois University Press, 19671987), 200.

3. Dewey, "Mexico's Educational Renaissance," 202.

4. Ernesto Meneses Morales, et al., Tendencias educativas oficiales en México: 1911-1934. (México: Centro de Estudios Educativos - Universidad Iberoamericana, 1998), 450. A pesar de que la literatura mantiene que Moisés Sáenz hizo un Ph.D. en Columbia, el registro de esta Universidad no tiene constancia de esta graduación.

5. Ibid.

6. Dewey, "Mexico's Educational Renaissance," 201.

7. Engracia Loyo, Gobiernos Revolucionarios y Educación Popular en México 1911-1928 (México, D.F.: El Colegio de México, 1998), 199.

8. Moisés Sáenz, Carapan (Morelia: Talleres Linotipográficos, 1969), 196.

9. Paul J. Vanderwood, "Explaining the Mexican Revolution," en: The Revolutionary Process in México: Essays on Political and Social Change, 1880-1940, O. Rodríguez y E. Jaime, (Los Angeles: UCLA Latin American Center, 1990), 97-114.

10. Lorenzo Meyer, Rafael Segovia y Alejandra Lajous, Historia de la Revolución Mexicana, 1928-1934, tomo 12. Los inicios de la institucionalización, (México: El Colegio de México, 1995), 178; Arnaldo Córdova, La ideología de la Revolución Mexicana: la formación del nuevo régimen (México, D.F.: ERA, 1972), 35-36.

11. Elsie Rockwell, "Schools of the Revolution: Enacting and Contesting State Forms in Tlaxcala, 1910-1930," en Everyday Forms of State Formation: Revolution and the Negotiation of Rule in Modern Mexico, eds. Gilbert Joseph y Daniel Nugent, (Durham and London: Duke University Press, 1994), 183-85; Elsie Rockwell, Hacer escuela, hacer estado: Las reformas posrevolucionarias vistas desde Tlaxcala, (Zamora: El Colegio de Michoacán, 2007); Ariadna Acevedo Rodrigo, "Struggles for Citizenship? Peasant Negotiation of Schooling in the Sierra Norte de Puebla, Mexico, 1921-1933," Bulletin of Latin American Research, 23, n. 2 (2004): 184.

12. Narciso Bassols, Memoria del Ramo de Educación Pública. Año de 1932, en Debates y propuestas de Narciso Bassols (Toluca: Ediciones del Estado de México, 1932), 46. 31.

13. Guillermo Palacios, La pluma y el arado (México, D.F.: El Colegio de México, 1999),

14. Nancy Leys Stephan, The Hour of Eugenics: Race, Gender, and Nation in Latin America, (Ithaca: Cornell University Press, 1991) 67-68, 73; Alexandra Minna Stern, "From Mestizophilia to Biotypology: Racialization and Science in Mexico 1920-1960," en Race and Nation in Modern Latin-America, N. Applebaum, et al (Chapel Hill: University of North Carolina Press, 2003), 187-210.

15. Gustave Le Bon, Leyes psicológicas de la evolución de los pueblos, (Madrid: Escobar, 1912); Carlos O. Bunge, Nuestra América, (Barcelona: s.n., 1903); Brita L. Horner, El carácter mexicano revelado por su literatura (México: SEP, 1925); Sáenz, Carapan, 90.

16. José Manuel Puig Cassauranc, Address delivered by Dr. José Manuel Puig Casauranc, Secretary of Public Education of Mexico, March 23, 1926, at Teachers College, Columbia University, New York, (México: SEP, 1926). SEP, Memoria de la Secretaría de Educación Pública 1934, vol. II (México: SEP, 1934), 539-40.

17. Enrique Corona Morfín, Al Servicio de la Escuela Popular, (México: SEP, 1963, 2. ed.), 46, 67 . 
18. Primitivo Álvarez, Informe del Jefe de la Misión, Villa Jiménez, Michoacán, AHSEP, Dirección de Misiones Culturales, Caja 42, Exp. 10. (1928).

19. Claude Fell, La influencia soviética en la educación Mexicana (1920-21), (México, D.F.: 1980).

20. Rafael Ramírez, "El gran período constructivo del movimiento educacional de México surgido de la revolucion" en: Obras completas de Rafael Ramírez (Jalapa, Veracruz: Gobierno del Estado de Veracruz, Direccion general de educación popular, 1968), tomo VIII, parte 2, 181.

21. Morfín, Al Servicio de la Escuela Popular, 30. Cita: Estudio acerca de la educación fundamental en México. México D.F.: SEP, Biblioteca Enciclopédica Popular, n. 183.

22. Francisco Arce Gurza, "En busca de una educación revolucionaria: 1924-1934," en Ensayos sobre historia de la educación en México, eds. Josefina Zoraida Vásquez, Dorothy Tanck de Estrada, Anne Staples y Francisco Arce Gurza (México: El Colegio de México, 1981), 183-84; Roland K. Goodenow, "The Progressive Educator and the Third World: A First Look at John Dewey," History of Education 19, n. 1 (March 1990), 23-40.

23. Palacios, La pluma y el arado, 66.

24. Graduada en 1910 por la Escuela Nacional de Maestros, en 1914, el gobierno de Carranza la pensionó para viajar por México y Estados Unidos para conocer nuevos métodos de enseñanza. En 1922 fue comisionada al Primer Congreso Panamericano de Mujeres, en Baltimore, y al Segundo Congreso Internacional de Educación Moral y Enseñanza de la Historia, en Ginebra. Allí conoció a Ferrière y a Rudolf Steiner a quien consideraba una de sus referencias intelectuales. En 1923 es nombrada Jefa del Departamento de Alfabetización (Campaña contra el analfabetismo) y, en 1930, inspectora de escuelas primarias en la ciudad de México. y en los siguientes años desempeñó de manera interina el cargo de Subjefa del Departamento de Enseñanza Primaria y Normal, e impartió cátedra de Bases pedagógicas en la Escuela Nacional de Maestros.

25. Francisco Larroyo, Historia comparada de la educación en México (México: Porrua, 1947).

26. Claude Fell, José Vasconcelos: Los años del águila, (México, UNAM, 1989), 30-32, 180; Adolphe Ferriere, L'école active, Les origines, (Ginebra: Forum, 1922) T. I., 103

27. Morales et al, Tendencias educativas oficiales en México, 390.

28. Salvador M. Lima y Marcelino Rentería, “La Escuela de la acción II," Educación 2, n. 5 (septiembre 1923), 294.

29. Anarquista-racionalista belga, defensor de la escuela nueva y miembro de la Ejecutiva de la Liga Internacional para la Educación Racional de la Infancia dirigida por F. Ferrer. Escribió La escuela nueva. Bosquejo de una educación basada sobre las leyes de la evolución humana (Barcelona: La Escuela Moderna, 1909). El eclecticismo reinante en el periodo se vislumbra, por ejemplo, en que Eslander fue considerado un "predecesor" por Romain Rolland, quien, a su vez, era uno de los autores favoritos de Vasconcelos.

30. António de Sena Faria de Vasconcelos (1880-1939) estudio derecho en Coimbra y en la Universidad Nueva de Bruselas, donde llegó a profesor (1903-4/14-15). En 1912 fundó la École Nouvelle Bièrges-Les-Wavre. Trabajó con Ferrière, quien alabó su escuela y le recomendó a los gobiernos de Bolivia y Cuba. Colaboró con Claparède en el Laboratorio de Psicología Experimental y el Bureau International des Écoles Nouvelles. Difundió la escuela nueva en Bolivia y Cuba, desde donde pudo influir en el interés mexicano por Ferrière. Vid: Vasconcelos, Faria de, Une École Nouvelle en Belgique, Adolphe Ferrière, preface. (Paris: Neuchatel \& Niestlé S. A. Editeurs, 1915). 
31. Patri era un maestro de escuela italiano que emigró a los USA y en 1928 escribió $L a$ Escuela del Porvenir, (Madrid: Imprenta Ciudad Lineal).

32. John Dewey, La escuela y la sociedad, Domingo Barnés traducción y prólogo, (Madrid: Tip. Artística, 1921?). 181.

33. Ramírez, "El gran período constructivo del movimiento educacional de México,"

34. Moisés Sáenz, Antología de Moisés Sáenz (México: Oasis, 1970), 17-18.

35. Sáenz, Carapan, 7 y ss, 102, 172-173, 194, 195.

36. S.n. "John Dewey," El maestro rural (1 de Junio de 1933), 31.

37. Para un entendimiento de Claparède y la discusión sobre Dewey en Ginebra vea Daniel Tröhler, "Langue as homeland: The Genevan reception of Pragmatism" in Inventing the modern self and John Dewey: Modernities and the traveling of pragmatism in Education ed. Tom Popkewitz (New York: Palgrave, 2006).

38. M. Ed. Claparède, "La pedagogía de John Dewey," Boletín de la Institución Libre de Enseñanza, XLVI, n. 753 (31 diciembre 1922), 353-561.

39. Experience and Nature, traducido por José Gaos (1948); Arts as Experience, traducido por Samuel Ramos (1949); Logic traducido por Eugenio Imaz (1950); Quest for Certainty, traducido por Eugenio Imaz (1951).

40. José Vasconcelos, "Examen de algunas doctrinas pedagógicas contemporáneas: El peligro de Dewey," en: Política Educativa de la Revolución (1910-1940, Guadalupe Montroy Huitrón (México: SEP, 1985), 71-78; José Vasconcelos, "Historia del pensamiento filosófico" (1935), en: Obras completas, Vol. 4 (México: Libreros mexicanos unidos, 1957), 1152. 1935).

41. José Vasconcelos, De Robinson a Odiseo: Pedagogía estructurativa (Madrid: Aguilar,

42. Tras completar la secundaria y la escuela normal en México, Manuel Barranco estudió durante seis años en los USA, dos en la escuela normal y cuatro en Columbia University (1910-14). Su tesis de maestría se tituló "Mexico, Its Educational Problems — Suggestions for Their Solution," Columbia Univ. Teachers College Contributions to Education, n. 73 (New York: Teachers College, 1915).

43. Antropólogo, arqueólogo y sociólogo, estudió con Boas en Columbia, donde se doctoró en 1921. Fue Director General de Antropología y Subsecretario de Educación Pública (1924-1925). Proponente de la incorporación en: Forjando patria: pro nacionalismo.

44. El futuro fundador y Director de Fondo de Cultura Económica y Rector de la Universidad Nacional se licenció en derecho (1925) y estudio economía en Harvard y Cornell. A mediados de los 20 visitó Columbia y acompañó a los Dewey a Vermont. 1947.09.12 (10567): Daniel Cosio Villegas to John Dewey. Number: 10567 en John Dewey, The Correspondence of John Dewey, 1871-1952 (II), (Carbondale: Southern Illinois University Press, 1999-2004).

45. Jay Martin, The education of John Dewey: a biography (New York: Columbia University Press, 2003), 341-45.

46. Dewey, The Later Works of John Dewey, 425-26.

47. Jesús Velasco, "Reading Mexico, Understanding the United States: American Transnational Intellectuals in the 1920s and 1990s," Journal of American History 86, n. 2 (1999), 641-67.

48. Jean Pierre Bastian, Breve Historia del Protestantismo en América Latina (México: Casa Unida de Publicaciones, 1986), 103-04. 
49. Rosa Bruno-Jofré, "To those in 'heathen darkness': Deweyan Democracy and Education in the American Interdenominatonal Configuration: The Case of the Committee on Cooperation in Latin America," en: Democracy and the Intersection of Religion and Traditions: The Readings of John Dewey's Understanding of Democracy and Education, Rosa Bruno-Jofré, Scott Johnston, Gonzalo Jover, y Daniel Tröhler (Kingston and Montreal: McGill-Queen's University Press, 2010); Vid. Rosa Bruno-Jofré, Methodist Education in Peru: Social gospel, politics, and American ideological and economic penetration, 1888-1930 (Waterloo, ON: Canadian Corporation for Studies in Religion - Wilfred Laurier University Press, 1988).

50. Donal Meyer, The Protestant Search for Political Realism, 1919-1941, 2nd ed. (Middletown, Connecticut: Wesleyan University Press, 1988), 137. Cit. en Bruno-Jofré, “To those in "heathen darkness."'

51. Daniel R. Miller, "Protestantism and Radicalism in Mexico from the 1860s to the 1930s," Fides et Historia, 40, n. 1 (Winter/Spring 2008), 43-66, 22. Vid. Jean-Pierre Bastian, Los disidentes: Sociedades protestantes y revolución en México, 1872-1911 (México D.F.: Fondo de Cultura Económica y El Colegio de México, 1989).

52. Miller, "Protestantism and Radicalism in Mexico," 22.

53. Ibid., 11.

54. Bruno-Jofré, "To those in 'heathen darkness."

55. Rosa María Torres Hernández, "Influencia de la teoría pedagógica de John Dewey en los periodos presidenciales de Plutarco Elías Calles y el Maximato, 1924-1934" (Tesis de Doctorado, UNAM, México, 1997).

56. Ramírez, Obras completas de Rafael Ramírez, Tomo V, 160-63.

57. Concepción Jiménez Alarcón, "Prólogo," Rafael Ramírez, Rafael Ramírez y la escuela Rural Mexicana (México, DF.: SEP, 1986), 14.

58. Meneses Morales, et al., Tendencias educativas oficiales en México, 389.

59. Ramírez, Obras completas de Rafael Ramirez, Tomo VIII, 13-14.

60. Ibid., Tomo IV, 43.

61. Omer Buyse, Méthodes américaines d'éducation générale et technique ([Charleroi]: H. Dunod \& E. Pinat, 1908), en: http://www.archive.org/details/ mthodesamericain01buysgoog.

62. Ramírez, Obras completas de Rafael Ramírez, Tomo IV, 28.

63. Herbert M. Kliebard, The Struggle for the American Curriculum, 1893-1958 (Boston: Routledge \& Kegan Paul, 1986), 45.

64. John Dewey, Democracy and Education (New York: The Free Press, 1944), 72-77.

65. Ramírez, Obras completas de Rafael Ramírez, Tomo V, 47, 49.

66. Palacios, La pluma y el arado, 70 y ss.

67. Ramírez, Obras completas de Rafael Ramírez, Tomo V, 64.

68. Ibid., 5, 47, 53, 49.

69. Sáenz, Carapan, 62.

70. Ramírez, Obras completas de Rafael Ramírez, Tomo V, 127, 129.

71. Ibid., 152-53.

72. Ibid., 145-47. 
73. Ibid., 161.

74. Sáenz, Carapan, 46.

75. Ramírez, Obras completas de Rafael Ramírez, Tomo V, 37, 40.

76. Sáenz, Carapan, 58.

77. Acevedo, "Struggles for Citizenship?," 188-89; Stephen E. Lewis, The Ambivalent Revolution, (Albuquerque: University of New Mexico Press, 2005), 52.

78. En las obras completas, que no están ordenadas cronológicamente, no se especifica la fecha de edición.

79. Rafael Ramírez, Los nuevos rumbos de la didáctica, Rafael Ramírez y la Escuela Rural Mexicana (México: SEP, 1986), 93-95.

80. John Dewey, The School and Society (Chicago, University of Chicago Press, 1899). 